\title{
Clubfoot: current concept of treatment
}

\author{
A. Andreacchio ${ }^{1}$, F. Alberghina ${ }^{2}$, S. Monforte ${ }^{1}$, A. Dimeglio ${ }^{3}$, F. Canavese ${ }^{4}$ \\ ${ }^{1}$ Vittore Buzzi Children's Hospital, Milano, Italy \\ ${ }^{2}$ Regina Margherita Children's Hospital, University of Torino, Torino, Italy \\ ${ }^{3}$ Clinique St. Roch, Montpellier, France \\ ${ }^{4}$ Lille University Center, Jeanne de Flandre Hospital, Nord-de-France University, Lille, France
}

\begin{abstract}
Introduction Idiopathic clubfoot (IC), also referred to as congenital talipes equinovarus, is one of the most common lower limb deformities observed in newborns, leading to significant functional impairment when left untreated. Early minimally invasive treatment has been praised as one of the most successful practice of modern pediatric orthopedics. This review aims to report current knowledge and controversies about clubfoot treatment. Material and methods We describe the main trends in clubfoot managing, identifying peculiarities, difficulties and prognostic factors related to the treatment. Results Many treatment techniques either conservative, surgical or hybrid have been used over the past decades. Based on good and excellent results during long-term follow-up, Ponseti method has been globally accepted by paediatric orthopaedic surgeons as standard method of treatment. However, some other conservative methods are still widely applied in the clinical setting, such as the French Physical Therapy method. Adherence to the bracing protocol is critical for the long-term success of the treatment, being a better predictor for relapse than severity of the deformity at birth. Conclusions Taking care of the manipulation and casting details by trained professionals, together with enhancing the child and patents' adherence to the brace, are essential for the success of conservative treatment. Surgery should be performed only when strictly needed, preferably on a "a la carte" approach.
\end{abstract}

Keywords: clubfoot, Ponseti, casting, French functional physiotherapy, hybrid method

\section{INTRODUCTION}

Idiopathic clubfoot (IC), also referred to as congenital talipes equinovarus, is one of the most common lower limb deformities observed in newborns, occurring at 1 to 2 per 1000 births. An awareness of the disorder has long been known since Egyptian age, and Hippocrates around 400 B.C., firstly postulated the main causing factor to be a mechanical pressure on the fetal foot.

Although the exact etiology of IC still remains unclear, a combination of genetic and environmental factors has been hypothesized to play a role in the pathogenesis of the deformity.

Congenital clubfoot deformity may occur as an isolated extremity defect, be syndromic, or associated with other congenital malformations and deformities such as spina bifida, DDH, constriction band syndrome, tibial hemimelia.

The etiology seems to be multifactorial including genetic, environmental and medical factors. The most common and clinically relevant factors include [1] family history, exposure during pregnancy to selective serotonin reuptake inhibitors (SSRI), amniocentesis, maternal/paternal smoking, maternal obesity (maternal BMI $>30$ measured pre-pregnancy/within first trimester) and gestational diabetes.

IC is a three-dimensional deformity of the foot which includes forefoot adductus, midfoot cavus, hindfoot varus, and ankle equinus and it is associated to calf amyotrophy.

Untreated and neglected clubfoot may cause a severe impairment of the lower extremity, resulting in a significant level of disability due to pain, stiffness, and gait disturbance.

Many treatment options either conservative, surgical or hybrid have been used over the past decades [2-4]. Historically, long periods of immobilization followed by extensive surgery such as soft tissue release or arthrodesis were the main treatments for IC. For example, one-stage posteromedial release (PMR) initially described by Codivilla, and then modified by Turco, included extensive soft tissue excision and capsulotomies and was largely performed three decades ago or so. However, high rates of painful and stiff feet with poor functional outcomes were reported [3].

Due to the controversial results of surgery and the modern anatomo-pathological findings, the treatment of IC has shifted to minimally invasive approaches. The most relevant example is the protocol developed by Ignacio Ponseti, Iowa, USA.

Based on good and excellent results during longterm follow-up, Ponseti method (PM) has been globally accepted by paediatric orthopedic surgeons as the standard conservative treatment. However, some other conservative methods are still widely applied in the clinical setting, such as the French Physical Therapy method (FPTM) and the recent "hybrid method" (HYM) [3].

This review aims to summarize the main trends in clubfoot managing, identifying peculiarities, difficulties and prognostic factors related to the treatment. 


\section{MATERIAL AND METHODS}

\section{Ponseti Method}

Starting from early 1990's, the PM, consisting of manipulation, casting, Achilles tenotomy in most cases, and bracing has become the most widely accepted protocol to treat IC during infancy $[5,6]$. Ponseti emphasized the importance of inversion of the foot in clubfoot pathology, considering the growth of the foot to be altered by the abnormal pressure of displaced navicular on talus ossification center [2].

His technique consists of two phases: treatment and maintenance. The first should be started as early as possible, within the first month of life. However, even a delay of few weeks does not significantly influence the number of casts needed, the incidence of cast-related or brace-related skin problems, parental brace compliance issues, or early relapse [7].

The physician performs gentle manipulation following three steps [8]: (a) Identify the head of the talus by palpation. (b) Supinate the forefoot to eliminate the cavus deformity and create physiological arch; (c) Abduct the forefoot in the plane of the sole using the lateral head of the talus as the fulcrum and maintaining the reduction of the cavus deformity.

Manipulation is followed by the application of an above-the-knee cast with the foot in the corrected position to renew every 5 to 7 days until the foot is abducted approximately $50^{\circ}$ from the frontal plane of the tibia.

Plaster of Paris (POP) and semirigid synthetic fiberglass (SRF) are the two most common casting materials used for IC. Specifically, POP has been advocated as the material of choice because of it is easily available, well moldable and cheap. However, over the years, limitations such as difficulty in removal, soiling and heaviness have been reported as exothermic reaction during plaster molding and skin lesions caused by the cast saw. SRF has thus been used as alternative casting material for its lighter weight, rapid application and removal, and because it needs no time lapse between removal and application of the new cast [7]. However, its higher cost and stiffness associated with reduced moldability compared to POP, have limited its use.

During casting, meticulous attention should be given to the technical details of manipulation, foot positioning and cast molding, especially in very small and short feet in which serial casting results more difficult and the risk of cast slippage higher [7,9].

The first deformity to be addressed is the cavus that is neutralized after the application of 2 or 3 casts by the simple positioning maneuver. Abduction of the forefoot while using the head of the talus as the fulcrum, following a vector of force parallel to the sole, results in the correction of the midfoot adduction deformity together with the hindfoot varus and the subtalar component of the equinus deformity.

After casting, 60 to $90 \%$ of infants need a percutaneous Achilles tendon tenotomy to correct the residual ankle equinus, that needs to be addressed after all other deformities have been completely corrected. If the foot dorsiflexion reaches $15^{\circ}$ (without midfoot breach), a tenotomy is not needed. The tenotomy can be performed as an outpatient procedure under local anesthesia or in the operation theatre under sedation or general anesthesia. The final cast is applied and removed after three weeks.

The maintenance phase starts after removal of the final cast and consists in wearing a foot abduction orthosis (FAO) to hold the feet in external rotation and dorsiflexion for 23 hours per day for 3 months. Bracing at night and during nap time is recommended until the age of 4 to 5 years old. Since there is no strict clinical surveillance, maintenance phase is probably the most challenging stage for the child and his family. Adherence to the bracing protocol is critical for the longterm success of the treatment, being a better predictor for relapse than severity of the deformity at birth [10].

Other potential causes for recurrence include the intrinsic contractile nature of the soft tissues, genetic and neuromuscular factors and casting imperfections [11].

Goldstein et al. reviewed 134 feet with a minimum of 3-year follow-up and found patients with poor adherence to foot abduction orthosis were 7.9 times more likely to need surgery. Female patients were 5.4 times more likely to need surgery than male patients. Dimeglio score at presentation and number of casts required prior to the start of a foot abduction brace were also predictive for treatment-resistant cases and need of additional surgery [4].

A fairly new clinical sign that may help to identify clubfeet resistant to the treatment is the "Samir-Adam sign" which indicates a relatively extended big toe compared to the lesser digits. The presence of this anomalous flexor digitorum accessorius longus muscle was observed in clubfeet resistant to casting [12].

Another risk factor for relapse that may be clinically identified is a reduced eversion muscle activity [13].

Overall, despite some loss of ankle motion and strength [14], clubfeet treated with the Ponseti method generally present good function, without affecting activities of daily living.

\section{French functional physiotherapy method and} hybrid method

The FPTM is based on serial manipulations and bandages to correct the various components of the deformity separately and in a specific order $[3,15]$. The main difference with the PM is the progressive and 
gradual correction by daily stretching, mainly focused on the medial side of the foot, and taping followed by splinting. The total duration of treatment is 1 to 3 months, and physiotherapist treats the patient five days per week. The technical skill and experience of the physiotherapist are crucial for the success of treatment, and probably this is the reason of significantly dissimilar outcomes reported in the literature [16]. Between 2 and 3 months of age, Achilles' tenotomy under general anesthesia is performed if one or more of the following elements persist: a) equinus; b) empty heel; c) posterior crease; or d) lack of divergence between talus and calcaneus on anterior-posterior (AP) and lateral foot radiographs.

After corrective phase in which calf massage, forefoot stretch, distraction, derotation, stimulation of evertors, hindfoot valgus, and dorsiflexion are performed, maintenance phase using splints starts. Another main stay of the method is the concept of "surgery à la carte". In this conception, surgery should be considered as a complementary procedure to nonoperative treatment, to perform in the most possible non-invasive manner, protecting ligaments, tendons and sheets from unneeded damages. For the same principle and to avoid fibrosis, all lengthening procedures should be performed only at the musculotendinous junction ("fractional lengthening") [15].

Overall, the protocol aims to achieve a pain-free supple plantigrade foot with as minimal surgery.

The HM proposed by Canavese et al. [3] adds manipulations and feet radiographs to the PM. It is called 'hybrid' because it merges together the strength of the PM (serial casting) and the FPTM (manipulations). In particular, the frequent manipulations allow to stimulate the foot at the end of each period of cast immobilization while the regular foot radiographs are essential to objectively evaluate the effect of the conservative treatment on the foot anatomy. In particular, radiographs detect any lack of divergence between talus and calcaneus that is sign of incomplete correction.

\section{Atypical Clubfoot}

Some clubfeet are more difficult to correct and may need a longer treatment. Excluding teratologic and syndromic deformities, some idiopathic clubfeet result unusually stiff presenting shortened and stubby shape of the forefoot, severe supination and equinus, and deep medial to lateral plantar crease at the midfoot [17]. These variants have been generally referred as 'complex' and 'atypical'. The 'four finger technique' has been specifically described by Ponseti to improve casting results in these cases. When manipulating, index and long fingers of both hands are positioned over the dorsum of the foot just anterior to the ankle joint. The index finger should rest over the posterior aspect of the lateral malleolus while the thumb of the same hand applies counterpressure over the lateral aspect of the talar head (4 upper). Once the maximum abduction of 30 degrees is achieved, both thumbs then push upwards to extend the metatarsals correcting the cavus and equinus (4 lower). Ponseti also recommended flexing the knee at least $110^{\circ}$ to avoid slippage, while other authors suggest moulding the upper anterior thigh region is a flat shape, if not actually concave, to prevent kicking of the knee [17]. After serial casting and tenotomy, a standard FAO, with the affected foot only externally rotated $40^{\circ}$, or an adequately moulded ankle foot orthosis is used.

\section{Treatment of relapse}

The most common complication in clubfoot treatment is relapse, occurring in up to $40 \%$ of patients who underwent initial successful treatment with the Ponseti method [11, 18]. Clinical signs of relapse may appear at different ages, and be present to varying degrees. The earliest sign includes some loss of ankle dorsiflexion compared to the previous examination. In older children, some elements of the deformity may distinctly reappear, such as forefoot adduction, cavus, heel varus, and a limitation of the foot abduction may be noted. During walking age, some gait disturbances may be present: increased contact of the lateral border of the forefoot during stance phase, varus of the heel, an inward deviation of the foot to the forward line of progression, and dynamic supination of the forefoot during swing phase [18].

During maintenance phase, a mild loss of dorsiflexion $\left(<10^{\circ}\right)$ may be successfully managed by increasing the duration of daily bracing and prescribing home stretching exercises. In case of loss of dorsiflexion $>10^{\circ}$ proper treatment should be started. In particular, a complete Ponseti protocol is effective to correct relapsed clubfeet up to 8 years of age [19], considering repeating Achilles tenotomy when dorsiflexion is less than $15^{\circ}$.

Preliminary casting is particularly indicated in non-passively correctible feet that would not benefit from a tibialis anterior tendon transfer (TATT) as first line of relapse treatment.

Ponseti recommended TATT in children over 2.5 years, transferring the tendon to third (lateral) cuneiform. This procedure is effective in patients presenting dynamic supination of the forefoot during swing phase with flexible and passively correctable feet [20].

If after casting $10^{\circ}$ of dorsiflexion cannot be still achieved, an Achilles lengthening procedure should be performed at the time of TATT.

Other procedures, such as combined cuboid cuneiform osteotomy, posterior ankle and subtalar release, extensive posteromedial release or correction by gradual distraction using the Ilizarov or hexapod frame, may be useful in selected patients with stiff, stubborn and neglected clubfeet [20]. 


\section{CONCLUSIONS}

In the last 3 to 4 decades, the primary treatment option of patients with clubfoot has shifted from extensive surgical release to minimally invasive approaches, mainly represented by the PM. Nowadays, this protocol is effective to correct all components of the deformity leading to a flexible and functional foot with minimal need of further surgery in most cases.

The numerous papers that have been published in the past several years highlight the success of clubfoot programs in terms of training, diffusion, low-cost and efficacy, also in low- and middle-income countries.

Taking care of the manipulation and casting details by trained professionals, together with enhancing the child and patents' adherence to bracing, are the keys of the success in the treatment, in which surgery should play a limited role based and on a "à la carte" approach. However, prospective randomized clinical trials are still needed to influence clinicians' and families' choices regarding best practicing.

No Source of financial support.

No Conflict of interest.

\section{REFERENCES}

1. Chen C., Kaushal N., Scher D.M., Doyle S.M., Blanco J.S., Dodwell E.R. Clubfoot Etiology: A Meta-Analysis and Systematic Review of Observational and Randomized Trials. J. Pediatr. Orthop.. 2018, vol. 38, no. 8, pp. e462-e469. DOI: 10.1097/BPO.0000000000001191.

2. Ponseti I.V. Treatment of congenital club foot. J. Bone Joint Surg. Am., 1992, vol. 74, no. 3, pp. 448-454.

3. Canavese F., Mansour M., Moreau-Pernet G., Gorce Y., Dimeglio A. The hybrid method for the treatment of congenital talipes equinovarus: preliminary results on 92 consecutive feet. J. Pediatr. Orthop. B, 2017, vol. 26, no. 3, pp. 197-203. DOI: 10.1097/BPB.0000000000000423.

4. Goldstein R.Y., Seehausen D.A., Chu A., Sala D.A., Lehman W.B. Predicting the need for surgical intervention in patients with idiopathic clubfoot. J. Pediatr. Orthop., 2015, vol. 35, no. 4, pp. 395-402. DOI: 10.1097/BPO.0000000000000282.

5. Owen R.M., Capper B., Lavy C. Clubfoot treatment in 2015: a global perspective. BMJ Glob. Health, 2018, vol. 3, no. 4, pp. e000852. DOI: 10.1136/ bmigh-2018-000852.

6. O'Shea R.M., Sabatini C.S. What is new in idiopathic clubfoot? Curr. Rev. Musculoskelet. Med., 2016, vol. 9, no. 4, pp. 470-477. DOI: 10.1007/ s12178-016-9375-2.

7. Zionts L.E., Sangiorgio S.N., Cooper S.D., Ebramzadeh E. Does Clubfoot Treatment Need to Begin As Soon As Possible? J. Pediatr. Orthop., 2016, vol. 36, no. 6, pp. 558-564. DOI: 10.1097/BPO.0000000000000514.

8. Dietz F.R., Noonan K. Treatment of Clubfoot Using the Ponseti Method. JBJS Essent. Surg. Tech., 2016, vol. 6, no. 3, pp. e28. DOI: 10.2106/JBJS. ST.14.00112.

9. Ko K.R., Shim J.S., Kim J.H., Cha Y.T. Difficulties During Ponseti Casting for the Treatment of Idiopathic Clubfoot. J. Foot Ankle Surg., 2020, vol. 59, no. 1, pp. 100-104. DOI: 10.1053/j.jfas.2019.07.022.

10. Alves C. Bracing in clubfoot: do we know enough? J. Child. Orthop., 2019, vol. 13, no. 3, pp. 258-264. DOI: 10.1302/1863- 2548.13.190069.

11. Van Praag V.M., Lysenko M., Harvey B., Yankanah R., Wright J.G. Casting Is Effective for Recurrence Following Ponseti Treatment of Clubfoot. J. Bone Joint Surg. Am., 2018, vol. 100, no. 12, pp. 1001-1008. DOI: 10.2106/JBJS.17.01049.

12. Shaheen S., Mursal H., Rabih M., Johari A. Flexor digitorum accessorius longus muscle in resistant clubfoot patients: introduction of a new sign predicting its presence. J. Pediatr. Orthop. B, 2015, vol. 24, no. 2, pp. 143-146. DOI: 10.1097/BPB.0000000000000129.

13. Gelfer Y., Dunkley M., Jackson D., Armstrong J., Rafter C., Parnell E., Eastwood D.M. Evertor muscle activity as a predictor of the mid-term outcome following treatment of the idiopathic and non-idiopathic clubfoot. Bone Joint J., 2014, vol. 96-B, no. 9, pp. 1264-1268. DOI: 10.1302/03 01-620X.96B9.33755.

14. Jeans K.A., Karol L.A., Erdman A.L., Stevens W.R. Jr. Functional Outcomes Following Treatment for Clubfoot: Ten-Year Follow-up. J. Bone Joint Surg. Am., 2018, vol. 100, no. 23, pp. 2015-2023. DOI: 10.2106/JBJS.18.00317.

15. Dimeglio A., Canavese F. The French functional physical therapy method for the treatment of congenital clubfoot. J. Pediatr. Orthop. B, 2012, vol. 21, no. 1, pp. 28-39. DOI: 10.1097/BPB.0b013e32834ee5f8.

16. He J.P., Shao J.F., Hao Y. Comparison of different conservative treatments for idiopathic clubfoot: Ponseti's versus non-Ponseti's methods. J. Int. Med. Res., 2017, vol. 45, no. 3, pp. 1190-1199. DOI: 10.1177/0300060517706801.

17. Van Bosse H.J.P. Challenging clubfeet: the arthrogrypotic clubfoot and the complex clubfoot. J. Child. Orthop., 2019 , vol. 13 , no. 3, pp. $271-281$. DOI: $10.1302 / 1863-2548.13 .190072$.

18. Hosseinzadeh P., Kelly D.M., Zionts L.E. Management of the Relapsed Clubfoot Following Treatment Using the Ponseti Method. J. Am. Acad. Orthop. Surg., 2017, vol. 25, no. 3, pp. 195-203. DOI: 10.5435/JAAOS-D-15-00624.

19. Dragoni M., Farsetti P., Vena G., Bellini D., Maglione P., Ippolito E. Ponseti Treatment of Rigid Residual Deformity in Congenital Clubfoot After Walking Age. J. Bone Joint Surg. Am., 2016, vol. 98, no. 20, pp. 1706-1712. DOI: 10.2106/JBJS.16.00053.

20. Eidelman M., Kotlarsky P., Herzenberg J.E. Treatment of relapsed, residual and neglected clubfoot: adjunctive surgery. J. Child. Orthop., 2019, vol. 13, no. 3, pp. 293-303. DOI: 10.1302/1863-2548.13.190079.

Received: 25.02 .2021

\section{Information about the authors:}

1. Antonio Andreacchio, M.D.,

Vittore Buzzi Children's Hospital, Milan, Italy,

Email: a.andreacchio@libero.it

2. Flavia Alberghina, M.D.,

Regina Margherita Children's Hospital, Turin, Italy,

University of Turin, Turin, Italy,

Email: falberghina@gmail.com

3. Sergio Monforte, M.D.,

Vittore Buzzi Children's Hospital, Milan, Italy,

Email: sergio.monforte@asst-fbf-sacco.it

4. Alain Dimeglio, M.D.,

Clinique St. Roch, Montpellier, France,

Email: alaindimeglio@wanadoo.fr

5. Federico Canavese, M.D., Ph.D.,

Lille University Center, Jeanne de Flandre Hospital, Nord-de-France University, Lille, France,

Email: canavese_federico@yahoo.fr 